\title{
Existence Results for a Perturbed Problem Involving Fractional Laplacians
}

\author{
Yan Hu \\ College of Mathematics and Econometrics, Hunan University, Changsha 410082, China \\ Correspondence should be addressed to Yan Hu; huyan1111@126.com
}

Received 25 October 2013; Accepted 20 February 2014; Published 27 March 2014

Academic Editor: Julio D. Rossi

Copyright (C) 2014 Yan Hu. This is an open access article distributed under the Creative Commons Attribution License, which permits unrestricted use, distribution, and reproduction in any medium, provided the original work is properly cited.

We extend the results of Cabre and Sire (2011) to show the existence of layer solutions of fractional Laplacians with perturbed nonlinearity $(-\Delta)^{s} u=b(x) f(u)$ in $\mathbb{R}$ with $s \in(0,1)$. Here $b$ is a positive periodic perturbation for $f$, and $-f$ is the derivative of a balanced well potential $G$. That is, $G \in C^{2, \gamma}$ satisfies $G(1)=G(-1)<G(\tau) \forall \tau \in(-1,1), G^{\prime}(1)=G^{\prime}(-1)=0$. First, for odd nonlinearity $f$ and for every $s \in(0,1)$, we prove that there exists a layer solution via the monotone iteration method. Besides, existence results are obtained by variational methods for $s \in(1 / 2,1)$ and for more general nonlinearities. While the case $s \leq 1 / 2$ remains open.

\section{Introduction}

We consider the classical fractional Laplacian

$$
(-\Delta)^{s} u=b(x) f(u) \quad \text { in } \mathbb{R}
$$

where $s \in(0,1)$ and

$$
(-\Delta)^{s} u=c_{s} \text { P.V. } \int_{\mathbb{R}} \frac{u(x)-u(y)}{|x-y|^{1+2 s}} d y .
$$

Here P.V. stands for the Cauchy principle value and $c_{s}$ is a positive constant depending only on $s$.

In the whole paper we suppose that $b \in\left(C^{1, \gamma} \cap L^{\infty}\right)(\mathbb{R})$ $(\gamma>\max \{0,1-2 s\})$ is a positive function and $b(x+1)=b(x)$ for every $x \in \mathbb{R}$.

It is well known that the fractional Laplacian is a nonlocal operator which can be localized by adding a variable

$$
\begin{gathered}
\operatorname{div}\left(y^{a} \nabla u(x, y)\right)=0 \quad \text { in } \mathbb{R}_{+}^{2}, \\
-d_{s} \lim _{y \downarrow 0^{+}} y^{a} \frac{\partial u}{\partial y}=b(x) f(u) \quad \text { on } \partial \mathbb{R}_{+}^{2}=\mathbb{R},
\end{gathered}
$$

where $a=1-2 s \in(-1,1),\left.u(x, y)\right|_{\partial \mathbb{R}_{+}^{2}}=u(x)$, and

$$
u(x, y)=p_{s} \int_{\mathbb{R}} \frac{y^{2 s}}{\left(y^{2}+z^{2}\right)^{(1+2 s) / 2}} u(x-z, 0) d z
$$

which is called the $s$-extension of $u(x, 0) . p_{s}$ is a positive constant multiplier depending only on $s$.

Obviously, (1) and (3) have their own variational structures. For nonlocal equation (1), its corresponding nonlocal energy functional on any open interval $I \subset \mathbb{R}$ is defined by

$$
\begin{aligned}
\mathscr{E}_{\text {non }}(u, I)= & \frac{1}{2} \iint_{I} \frac{|u(x)-u(y)|^{2}}{|x-y|^{1+2 s}} d y d x \\
& +\int_{I} \int_{\mathbb{R} \backslash I} \frac{|u(x)-u(y)|^{2}}{|x-y|^{1+2 s}} d y d x \\
& +\int_{I} b(x) G(u) d x
\end{aligned}
$$

with prescribed boundary data outside of $I$.

As for elliptic equation (3), its local energy functional is given by

$$
\mathscr{E}_{\mathrm{loc}}(u, \Omega)=\frac{d_{s}}{2} \int_{\Omega} y^{a}|\nabla u|^{2} d x d y+\int_{\partial \Omega \cap \partial \mathbb{R}_{+}^{2}} b(x) G(u) d x
$$

with $\Omega \subset \mathbb{R}_{+}^{2}$ an open domain. Here $G$ is the primitive function of $-f$. The details for the fractional Laplacian can be seen in $[1-6]$. 
In view of the De Giorgi conjecture [7-9], Cabré and Sire [1-3] consider layer solutions of the autonomous equation

$$
(-\Delta)^{s} u=f(u) \quad \text { in } \mathbb{R} .
$$

The layer solution of (7) is a strictly increasing solution with limits \pm 1 at $\pm \infty$. In $[2,3]$ the necessary and sufficient conditions for existence of layer solutions were presented by a Hamiltonian equality and a Modica-type estimate via variational methods, that is, $G^{\prime}(1)=G^{\prime}(-1)=0$ and $G(1)=$ $G(-1)<G(\tau)$ for every $\tau \in(-1,1)$. In other words, (7) is an Allen-Cahn-type equation.

Equation (7) does not depend on the variable $x$ explicitly; layer solutions were proved to be strictly increasing and are the unique local minimizer by the sliding method. Furthermore, if $f$ is odd and $f^{\prime}( \pm 1)<0$, the layer solution is proved odd symmetric about some point where it equals zero. Many properties about the fractional Laplacian were established such as the regularity, Hopf lemma, harnack, and maximum principle; for details see $[2,3]$.

We give the definition of layer solutions of (1).

Definition 1. A function $u \in\left(C_{\mathrm{loc}}^{\beta} \cap L^{\infty}\right)(\mathbb{R})(0<\beta<1)$ is said to be a layer solution of (1) if it satisfies

$$
\begin{gathered}
(-\Delta)^{s} u=b(x) f(u) \quad \text { in } \mathbb{R} \\
u \longrightarrow \pm 1 \quad \text { as } x \longrightarrow \pm \infty
\end{gathered}
$$

Different from (7), (1) depends on the variable $x$ explicitly; the sliding method cannot be used anymore and layer solutions of (1) have no monotonicity. Therefore, methods in $[2,3]$ for existence of layer solutions cannot be used here; our problem becomes complicated. In addition, the fractional Laplacian is a nonlocal case. All these cause some difficulties for existence results of layer solutions of (1).

In our previous work [10], assuming that $f$ was odd and $b$ was even, we proved the existence of layer solutions of (1) by variational methods and a Liouville theorem for $s \in[1 / 2,1)$. The restriction on $s$ was due to no Liouville-type conclusion for $0<s<1 / 2$. We also obtained a Hamiltonian equality and asymptotic estimates as $x \rightarrow \pm \infty$ for layer solutions. Furthermore, the asymptotic behavior of layer solutions as $s \uparrow 1$ is also investigated.

In this paper, we still study existence results of layer solutions of (1). At first we assume that $f$ is odd and $b$ is even. For this case, we mainly consider the $s$-extension of $u$; layer solutions are constructed by the subsupersolution technique for all $s \in(0,1)$.

Theorem 2. Suppose that $f \in C^{1, \gamma}(\mathbb{R})(\gamma>\max \{0,1-2 s\})$ and

(1) $f(-\tau)=-f(\tau)$ for every $\tau \in[-1,1]$;

(2) $f(1)=f(-1)=f(0)=0, f \geq 0$ in $[0,1]$ and $f \leq 0$ in $[-1,0]$.

Obviously, if $G(u)=\int_{u}^{-1} f(s) d s$,

$G(1)=G(-1)<G(\tau) \quad$ for $\tau \in(-1,1) ; G^{\prime}( \pm 1)=0$.
If $b$ is even, then there exists a layer solution $u \in$ $C^{2, \alpha}(\mathbb{R})(0<\alpha<1)$ of (1) which is also odd,

$$
\begin{gathered}
\left(-\partial_{x x}\right)^{s} u=b(x) f(u) \quad \text { in } \mathbb{R}, \\
u \longrightarrow \pm 1 \quad \text { as } x \longrightarrow \pm \infty .
\end{gathered}
$$

For more general nonlinearities $f$ and $s \in(1 / 2,1)$, existence results are proved by variational methods and a careful energy comparison. This part needs some tricks.

Theorem 3. Let $s \in(1 / 2,1)$. Let $G \in C^{2, \gamma}(\mathbb{R})(\gamma>\max \{0,1-$ $2 s\})$ be the primitive function of $-f$ and

(a) $G(1)=G(-1)<G(\tau)$ for $\tau \in(-1,1), G^{\prime}(1)=$ $G^{\prime}(-1)=0$;

(b) $G^{\prime \prime}(1)>0$ and $G^{\prime \prime}(-1)>0$.

Then, there exists a layer solution $u \in C^{2, \alpha}(\mathbb{R})$ of $(1)$ for some $0<\alpha<1$,

$$
\begin{gathered}
\left(-\partial_{x x}\right)^{s} u=b(x) f(u) \quad \text { in } \mathbb{R}, \\
u \longrightarrow \pm 1 \quad \text { as } x \longrightarrow \pm \infty .
\end{gathered}
$$

Remark 4. From the assumption above, there exists a $0<$ $\delta<1 / 2$ such that $G$ is strictly increasing in $[-1,-1+\delta]$ and decreasing in $[1-\delta, 1], G(s)>\min \{G(1-\delta), G(-1+\delta)\}$ for $s \in(-1+\delta, 1-\delta)$.

For $s \leq 1 / 2$, the global energy may be infinite. The desired asymptotic behavior of layer solutions at infinity, that is, $u \rightarrow$ \pm 1 as $x \rightarrow \pm \infty$, cannot be obtained. This case is open.

The paper is organized as follows. In Section 2, we prove existence results of layer solutions of (1) under odd assumptions of $f$. Section 3 is devoted to the Proof of Theorem 3. Finally, we give some results about regularity and gradient estimates and a Hopf lemma about (1) and (3) in the appendix.

For convenience, we give some notations.

One has

$$
\bar{b}=\max _{\mathbb{R}} b(x), \quad \underline{b}=\min _{\mathbb{R}} b(x) .
$$

Let $u \in H^{s}(I)$,

$$
\|u\|_{H^{s}(I)}=\|u\|_{\dot{H}^{s}(I)}+\left(\int_{I} u^{2} d x\right)^{1 / 2},
$$

where

$$
\begin{aligned}
\|u\|_{\dot{H}^{s}(I)}= & \frac{1}{2} \iint_{I} \frac{|u(x)-u(y)|^{2}}{|x-y|^{1+2 s}} d y d x \\
& +\int_{I} \int_{\mathbb{R} \backslash I} \frac{|u(x)-u(y)|^{2}}{|x-y|^{1+2 s}} d y d x
\end{aligned}
$$

is a seminorm.

\section{Odd Nonlinearities}

The main aim of this section is to solve existence problem for odd nonlinearities. We assume that $f$ satisfies assumptions 
in Theorem 2. For example, $f(u)=u-u^{3}$, the nonlinearity of Allen-Cahn equation satisfies this condition. Here we construct a layer solution of (1) by the subsupersolution method. For this purpose, let us recall results in [3].

Lemma 5. Let $f \in C^{1, \gamma}(\mathbb{R})(\gamma>\max \{0,1-2 s\})$, where $s \in$ $(0,1)$, such that $f=-G^{\prime}$. Then there exists a solution $u$ of

$$
\left(-\partial_{x x}\right)^{s} u=f(u) \quad \text { in } \mathbb{R}
$$

such that $u^{\prime}>0$ in $R$ and $\lim _{x \rightarrow \pm \infty} u= \pm 1$ if and only if

$$
G^{\prime}(1)=G^{\prime}(-1)=0, \quad G>G(1)=G(-1) \quad \text { in }(-1,1) \text {. }
$$

If in addition $f^{\prime}(-1)<0$ and $f^{\prime}(1)<0$, then this solution is unique up to translations.

As a consequence, if $f$ is odd and $f^{\prime}( \pm 1)<0$, then the solution is odd with respect to some point. That is, $u(x+b)=$ $-u(-x+b)$ for some $b \in \mathbb{R}$.

Remark 6. Denote $u^{*}$ to be the odd solution of (15) such that $u^{*}(0)=0$. Define $\bar{u}(x)=u^{*}\left(\bar{b}^{1 / 2 s} x\right)$ and $\underline{u}(x)=u^{*}\left(\underline{b}^{1 / 2 s} x\right)$; they are a supersolution and a subsolution of $(1)$ in $(0, \infty)$. Indeed, by simple calculations,

$$
\begin{aligned}
(-\Delta)^{s} \bar{u} & =(-\Delta)^{s} u^{*}\left(\bar{b}^{1 / 2 s} x\right)=\bar{b} f\left(u^{*}\left(\bar{b}^{1 / 2 s} x\right)\right) \\
& \geq b(x) f(\bar{u}) \quad \text { in }(0, \infty), \\
(-\Delta)^{s} \underline{u} & =(-\Delta)^{s} u^{*}\left(\underline{b}^{1 / 2 s} x\right) \\
& =\underline{b} f\left(u^{*}\left(\underline{b}^{1 / 2 s} x\right)\right) \leq b(x) f(\underline{u}) \text { in }(0, \infty) .
\end{aligned}
$$

Furthermore, $\bar{u}>\underline{u}$ in $(0, \infty)$ by monotonicity of $u^{*}$ and the fact that $\bar{b}>\underline{b}$.

In order to prove Theorem 2, we give the following two lemmas.

Lemma 7. Let $\bar{u}(\cdot, \cdot)$ and $\underline{u}(\cdot, \cdot)$ be the s-extensions of $\bar{u}(\cdot, 0)$ and $\underline{u}(\cdot, 0)$, respectively, in $\mathbb{R}_{+}^{2}$; then $\bar{u}>\underline{u}$ for $x>0$ and $y \geq 0$. Furthermore, one has

$$
\begin{gathered}
\operatorname{div}\left(y^{a} \nabla \bar{u}\right)=0 \quad \text { in } \mathbb{R}_{+}^{2} \cap\{x>0\}, \\
-d_{s} \lim _{y \downarrow 0^{+}} y^{a} \frac{\partial \bar{u}}{\partial y} \geq b(x) f(\bar{u}) \quad \text { on } \partial \mathbb{R}_{+}^{2} \cap\{x>0\}, \\
\operatorname{div}\left(y^{a} \nabla \underline{u}\right)=0 \quad \text { in } \mathbb{R}_{+}^{2} \cap\{x>0\}, \\
-d_{s} \lim _{y \downarrow 0^{+}} y^{a} \frac{\partial \underline{u}}{\partial y} \leq b(x) f(\underline{u}) \quad \text { on } \partial \mathbb{R}_{+}^{2} \cap\{x>0\} .
\end{gathered}
$$

Proof. For every $u(\cdot, 0) \in\left(C \cap L^{\infty}\right)(\mathbb{R}), u(x, y)(y>0)$, the $s$-extension of $u(x, 0)$ can be expressed by

$$
\begin{aligned}
u(x, y) & =p_{s} \int_{\mathbb{R}} \frac{y^{2 s}}{\left(z^{2}+y^{2}\right)^{(1+2 s) / 2}} u(x-z, 0) d z \\
& =p_{s} \int_{\mathbb{R}} \frac{1}{\left\{z^{2}+1\right\}^{(1+2 s) / 2}} u(x-y z, 0) d z .
\end{aligned}
$$

If $v(x, 0)=u(a x, 0)$, for some $a \in \mathbb{R}$,

$$
\begin{aligned}
v(x, y) & =p_{s} \int_{\mathbb{R}} \frac{1}{\left(z^{2}+1\right)^{(1+2 s) / 2}} v(x-y z, 0) d z \\
& =p_{s} \int_{\mathbb{R}} \frac{1}{\left(z^{2}+1\right)^{(1+2 s) / 2}} u(a x-a y z, 0) d z \\
& =u(a x, a y) .
\end{aligned}
$$

Thus, $\bar{u}(x, y)=u^{*}\left(\bar{b}^{1 / 2 s} x, \bar{b}^{1 / 2 s} y\right)$ and $\underline{u}(x, y)=u^{*}\left(\underline{b}^{1 / 2 s} x\right.$, $\left.\underline{b}^{1 / 2 s} y\right)$.

$$
\text { In addition, if } u(-x)=-u(x) \text {, }
$$

$$
\begin{aligned}
& u(x, y)=p_{s} \int_{\mathbb{R}} \frac{1}{\left(z^{2}+1\right)^{(1+2 s) / 2}} u(x-y z, 0) d z \\
& =p_{s} \int_{-\infty}^{x / y} \frac{1}{\left(z^{2}+1\right)^{(1+2 s) / 2}} u(x-y z, 0) d z \\
& +p_{s} \int_{x / y}^{+\infty} \frac{1}{\left(z^{2}+1\right)^{(1+2 s) / 2}} u(x-y z, 0) d z \\
& =p_{s} \int_{-\infty}^{0} \frac{1}{\left\{(z+(x / y))^{2}+1\right\}^{(1+2 s) / 2}} u(-y z, 0) d z \\
& +p_{s} \int_{0}^{+\infty} \frac{1}{\left\{(z+(x / y))^{2}+1\right\}^{(1+2 s) / 2}} u(-y z, 0) d z \\
& =p_{s} \int_{0}^{+\infty}\left\{\frac{1}{\left[((x / y)-z)^{2}+1\right]^{(1+2 s) / 2}}\right. \\
& \left.-\frac{1}{\left[((x / y)+z)^{2}+1\right]^{(1+2 s) / 2}}\right\} \\
& \times u(y z, 0) d z .
\end{aligned}
$$


Thus, for $x>0$ and $y>0$,

$$
\begin{aligned}
& \bar{u}(x, y)-\underline{u}(x, y) \\
& =p_{s} \int_{0}^{\infty}\left\{\frac{1}{\left[((x / y)-z)^{2}+1\right]^{(1+2 s) / 2}}\right. \\
& \left.-\frac{1}{\left[((x / y)+z)^{2}+1\right]^{(1+2 s) / 2}}\right\} \\
& \quad \times\left\{u^{*}\left(\bar{b}^{1 / 2 s} y z, 0\right)-u^{*}\left(\underline{b}^{1 / 2 s} y z, 0\right)\right\} d z \\
& >0
\end{aligned}
$$

since $u^{*}$ is increasing.

Equation (18) is the consequence of direct calculations; the proof of this lemma is complete.

Now let $\Omega \subset \mathbb{R}_{+}^{2}$ be a Lipschitz bounded domain; define the energy functional

$$
\begin{aligned}
\widetilde{\mathscr{E}}_{\mathrm{loc}}(w, \Omega)= & \frac{d_{s}}{2} \int_{\Omega} y^{a}|\nabla w|^{2} d x d y \\
& +\frac{A}{2} \int_{\partial \Omega \cap \partial \mathbb{R}_{+}^{2}} w^{2} d x-\int_{\partial \Omega \cap \partial \mathbb{R}_{+}^{2}} g(x) w d x,
\end{aligned}
$$

where $A>0$ is a constant and $g \in C^{1, \gamma}(\mathbb{R}) \cap L^{\infty}(\mathbb{R})(\gamma>$ $\max \{0,1-2 s\})$ is a nonnegative function.

Lemma 8. Given $w_{0} \in C^{2, \alpha}\left(\mathbb{R}_{+}^{2}\right)$ for some $0<\alpha<1$. Let $\Gamma=\left\{w \in H^{1}\left(y^{a}, \Omega\right), w=w_{0}\right.$ on $\left.\overline{\partial \Omega \cap \mathbb{R}_{+}^{2}}\right\}$. Then there exists a minimizer $w \in \Gamma$ such that $w \in C^{0, \beta}(\bar{\Omega})$ for some $0<\beta<1$ and satisfies the Euler-Lagrange equation:

$$
\begin{gathered}
\operatorname{div}\left(y^{a} \nabla w\right)=0 \quad \text { in } \Omega, \\
-d_{s} \lim _{y \downarrow 0^{+}} y^{a} \frac{\partial w}{\partial y}+A w=g(x) \quad \text { on } \partial \Omega \cap \partial \mathbb{R}_{+}^{2}, \\
w=w_{0} \text { on } \overline{\partial \Omega \cap \mathbb{R}_{+}^{2}} .
\end{gathered}
$$

Proof. One has

$$
\begin{aligned}
\widetilde{\mathscr{E}}_{\mathrm{loc}} & (w, \Omega) \\
= & \frac{1}{2} \int_{\Omega} y^{a}|\nabla w|^{2}+\frac{A}{2} \int_{\partial \Omega \cap \partial \mathbb{R}_{+}^{2}} w^{2}-\int_{\partial \Omega \cap \partial \mathbb{R}_{+}^{2}} g(x) w \\
\geq & \frac{1}{2} \int_{\Omega} y^{a}|\nabla w|^{2}+\frac{A}{2} \int_{\partial \Omega \cap \partial \mathbb{R}_{+}^{2}} w^{2}-\frac{A}{4} \int_{\partial \Omega \cap \partial \mathbb{R}_{+}^{2}} w^{2} \\
& -\frac{1}{A}\|g\|_{L^{\infty}}^{2} \cdot\left|\partial \Omega \cap \partial \mathbb{R}_{+}^{2}\right| \\
= & \frac{1}{2} \int_{\Omega} y^{a}|\nabla w|^{2}+\frac{A}{4} \int_{\partial \Omega \cap \partial R_{+}^{2}} w^{2} \\
& -\frac{1}{A}\|g\|_{L^{\infty}}^{2} \cdot\left|\partial \Omega \cap \partial \mathbb{R}_{+}^{2}\right| .
\end{aligned}
$$

Obviously, $w-w_{0} \in H^{1}\left(y^{a}, \Omega\right)$; we now extend it by zeroes outside of $\Omega$ in $\mathbb{R}_{+}^{2}, w-w_{0} \in H^{1}\left(y^{a}, \mathbb{R}_{+}^{2}\right)$ and by the trace theorem and compact imbedding theorem [11-13], $w-w_{0} \in$ $H^{s}(\mathbb{R}) \hookrightarrow \hookrightarrow L^{p}(\mathbb{R})$ for every $1 \leq p<\infty$. Therefore, $\widetilde{E}$ is well defined, bounded below, and coercive in $\Gamma$; there is a minimizer denoted again by $w$. By variational calculations,

$$
\begin{gathered}
\operatorname{div}\left(y^{a} \nabla w\right)=0 \quad \text { in } \Omega, \\
-d_{s} \lim _{y \downarrow 0^{+}} y^{a} \frac{\partial w}{\partial y}+A w=g(x) \quad \text { on } \partial \Omega \cap \partial \mathbb{R}_{+}^{2}, \\
w=w_{0} \quad \text { on } \overline{\partial \Omega \cap \mathbb{R}_{+}^{2}}
\end{gathered}
$$

in weak sense. Furthermore, $w \in C^{0, \beta}(\bar{\Omega})$ for some $0<\beta<1$ by regularity discussion.

Now we come to prove Theorem 2.

Proof of Theorem 2. By Lemma 7, $\bar{u}=u^{*}\left(\bar{b}^{1 / 2 s} x, \bar{b}^{1 / 2 s} y\right)$ and $\underline{u}=u^{*}\left(\underline{b}^{1 / 2 s} x, \underline{b}^{1 / 2 s} y\right)$ are a supersolution and a subsolution of (3) in $\mathbb{R}_{+}^{2} \cap\{x>0\}$, respectively. Let $\Omega_{R}=(0, R) \times(0, R)$ and $u_{0}=\bar{u}$. Consider the mixed-boundary problem

$$
\begin{gathered}
\operatorname{div}\left(y^{a} \nabla u_{1}\right)=0 \quad \text { in } \Omega_{R}, \\
-d_{s} \lim _{y \downarrow 0^{+}} y^{a} \frac{\partial u_{1}}{\partial y}+A u_{1}=b(x) f\left(u_{0}\right)+A u_{0} \\
\text { on } \partial \Omega_{R} \cap \partial \mathbb{R}_{+}^{2}, \\
u_{1}=\bar{u} \quad \text { on } \overline{\partial \Omega_{R} \cap \mathbb{R}_{+}^{2}},
\end{gathered}
$$

where $A>\max \left\{0, \bar{b} \max _{[0,1]}\left(-f^{\prime}\right)\right\}$. By Lemma 8 , there is a solution $u_{1} \in C^{0, \beta}\left(\bar{\Omega}_{R}\right)$ for some $0<\beta<1$, and further $u_{1}>0$ in $\Omega_{R}$ by the strong maximum principle and Hopf lemma.

Denote $w=u_{1}-u_{0}$,

$$
\begin{gathered}
\operatorname{div}\left(y^{a} \nabla w\right)=0 \quad \text { in } \Omega_{R}, \\
-d_{s} \lim _{y \downarrow 0^{+}} y^{a} \frac{\partial w}{\partial y}+A w \leq 0 \quad \text { on } \partial \Omega_{R} \cap \partial \mathbb{R}_{+}^{2}, \\
w=0 \quad \text { on } \overline{\partial \Omega_{R} \cap \mathbb{R}_{+}^{2}} .
\end{gathered}
$$

Again, by the strong maximum principle and Hopf lemma, $u_{1}<u_{0}$ in $\Omega_{R}$.

Now we start the iteration procedure. Given $u_{n}$ and $u_{n-1}$ such that $0 \leq u_{n} \leq u_{n-1} \leq 1$ in $\Omega_{R}$, the problem

$$
\begin{gathered}
\operatorname{div}\left(y^{a} \nabla u_{n+1}\right)=0 \quad \text { in } \Omega_{R}, \\
-d_{s} \lim _{y \downarrow 0^{+}} y^{a} \frac{\partial u_{n+1}}{\partial y}+A u_{n+1}=b(x) f\left(u_{n}\right)+A u_{n} \\
\text { on } \partial \Omega_{R} \cap \partial \mathbb{R}_{+}^{2}, \\
u_{n+1}=\bar{u} \quad \text { on } \overline{\partial \Omega_{R} \cap \mathbb{R}_{+}^{2}}
\end{gathered}
$$


has a solution $u_{n+1} \in C^{0, \beta}\left(\bar{\Omega}_{R}\right)$ for some $0<\beta<1$ by Lemma 8 and $u_{n+1}>0$ in $\Omega_{R}$ by the strong maximum principle and Hopf lemma.

Let $w=u_{n+1}-u_{n}$,

$$
\begin{gathered}
\operatorname{div}\left(y^{a} \nabla w\right)=0 \quad \text { in } \Omega_{R}, \\
-d_{s} \lim _{y \downarrow 0^{+}} y^{a} \frac{\partial w}{\partial y}+A w \\
=\left\{b(x) \frac{f\left(u_{n}\right)-f\left(u_{n-1}\right)}{u_{n}-u_{n-1}}+A\right\}\left(u_{n}-u_{n-1}\right) \\
\text { on } \partial \Omega_{R} \cap \partial \mathbb{R}_{+}^{2}, \\
w=0 \quad \text { on } \overline{\partial \Omega_{R} \cap \mathbb{R}_{+}^{2} .}
\end{gathered}
$$

Again by the strong maximum principle and Hopf lemma, $u_{n+1}<u_{n}$ in $\Omega_{R}$.

Assume that $u_{n} \geq \underline{u}$ in $\Omega_{R}$ for some $n \in Z^{+}$. Let $w=$ $u_{n+1}-\underline{u}$,

$$
\begin{gathered}
\operatorname{div}\left(y^{a} \nabla w\right)=0 \quad \text { in } \Omega_{R}, \\
-d_{s} \lim _{y \downarrow 0^{+}} y^{a} \frac{\partial w}{\partial y}+A w \\
=\left\{b(x) \frac{f\left(u_{n}\right)-f(\underline{u})}{u_{n}-\underline{u}}+A\right\}\left(u_{n}-\underline{u}\right) \\
\text { on } \partial \Omega_{R} \cap \partial \mathbb{R}_{+}^{2}, \\
w=\bar{u}-\underline{u} \geq 0 \quad \text { on } \overline{\partial \Omega_{R} \cap \mathbb{R}_{+}^{2}} ;
\end{gathered}
$$

$u_{n+1}>\underline{u}$ in $\Omega_{R}$ by maximum principle and Hopf lemma.

Thus there is a sequence $\left\{u_{n}\right\}, \bar{u}=u_{0}>u_{1}>u_{2}>\cdots>$ $u_{n}>\cdots>\underline{u}, u_{n} \rightarrow u_{R}$ by monotone convergence theorem and

$$
\begin{gathered}
\operatorname{div}\left(y^{a} \nabla u_{R}\right)=0 \quad \text { in } \Omega_{R}, \\
-d_{s} \lim _{y \downarrow 0^{+}} y^{a} \frac{\partial u_{R}}{\partial y}=b(x) f\left(u_{R}\right) \quad \text { on } \partial \Omega_{R} \cap \partial \mathbb{R}_{+}^{2}, \\
u_{R}=\bar{u} \quad \text { on } \overline{\partial \Omega_{R} \cap \mathbb{R}_{+}^{2}} ;
\end{gathered}
$$

$\underline{u} \leq u_{R} \leq \bar{u}$ in $\Omega_{R}$ and $\left\|u_{R}\right\|_{C^{0, \beta}\left(\bar{\Omega}_{R}\right)} \leq C$ where $C$ does not depend on $R$ by regularity discussion.

Up to a subsequence $u_{R} \rightarrow u$ in $C_{\text {loc }}^{0}\left(\mathbb{R}_{+}^{2} \cap\{x>0\}\right)$, $y^{a}\left(u_{R}\right)_{y} \rightarrow y^{a} u_{y}$ in $C_{\mathrm{loc}}^{0}\left(\overline{\mathbb{R}_{+}^{2}} \cap\{x>0\}\right)$ as $R \rightarrow \infty$, and

$$
\begin{gathered}
\operatorname{div}\left(y^{a} \nabla u\right)=0 \quad \text { in } \mathbb{R}_{+}^{2} \cap\{x>0\} \\
-d_{s} \lim _{y \downarrow 0^{+}} y^{a} \frac{\partial u}{\partial y}=b(x) f(u) \quad \text { on } \partial \mathbb{R}_{+}^{2} \cap\{x>0\}, \\
u(0, y)=0 \quad \text { for } y \geq 0
\end{gathered}
$$

$\underline{u}<u<\bar{u}$ in $\overline{\mathbb{R}_{+}^{2}} \cap\{x>0\}$ by the strong maximum principle and Hopf lemma. Therefore $u \rightarrow 1$ as $x \rightarrow \infty$ from asymptotic behavior of $\bar{u}$ and $\underline{u}$ as $x \rightarrow \infty$.
We define $u(x, y)=-u(-x, y)$ for $x<0$ and $y \geq 0$; then

$$
\begin{gathered}
\operatorname{div}\left(y^{a} \nabla u\right)=0 \quad \text { in } \mathbb{R}_{+}^{2}, \\
-d_{s} \lim _{y \downarrow 0^{+}} y^{a} \frac{\partial u}{\partial y}=b(x) f(u) \quad \text { on } \partial \mathbb{R}_{+}^{2},
\end{gathered}
$$

and $u \rightarrow \pm 1$ as $x \rightarrow \pm \infty$. The desired solution is achieved. We complete the proof.

\section{Nonsymmetric Nonlinearities}

In this section, we deal with more general situations; that is, $G \in C^{2, \gamma}(\mathbb{R})(\gamma>\max \{0,1-2 s\})$ :

(1) $G^{\prime}(1)=G^{\prime}(-1)=0, G(1)=G(-1)<G(\tau)$ for $\tau \epsilon$ $(-1,1)$;

(2) $G^{\prime \prime}(1)>0, G^{\prime \prime}(-1)>0$.

For simplicity we can assume that $G( \pm 1)=0$ by adding a constant.

Consider the nonlocal energy functional defined on an open interval $I \subset \mathbb{R}$,

$$
\begin{aligned}
\mathscr{E}_{\text {non }}(w, I)= & \frac{1}{2} \iint_{I} \frac{|w(x)-w(y)|^{2}}{|x-y|^{1+2 s}} d y d x \\
& +\int_{I} \int_{\mathbb{R} \backslash I} \frac{|w(x)-w(y)|^{2}}{|x-y|^{1+2 s}} d y d x \\
& +\int_{I} b(x) G(w) d x,
\end{aligned}
$$

where $w \in H^{s}(I)$ with prescribed boundary data outside of $I$.

Define a nondecreasing competitor

$$
h(x)= \begin{cases}1 & \text { if }|x| \geq 1 \\ x & \text { if }|x|<1\end{cases}
$$

If $|I|>4$, by direct calculations,

$$
\mathscr{E}_{\text {non }}(h, I) \leq \begin{cases}C_{s}\left(1+|I|^{1-2 s}\right) & \text { if } s \in\left(0, \frac{1}{2}\right), \\ C_{s}(1+\log |I|) & \text { if } s=\frac{1}{2}, \\ C_{s} & \text { if } s \in\left(\frac{1}{2}, 1\right),\end{cases}
$$

where $C_{s}$ is a positive constant depending only on $s$ (see [6]). In order to prove Theorem 3 , we first give several lemmas.

Lemma 9. Let $I=(a, b)$ with $b-a>4$. Let $\Gamma_{I}=\left\{w \in H^{s}(I)\right.$, $w=-1$ for $x \leq a$ and $w=1$ for $x \geq b,|w| \leq 1\}$. Then, for every $\in(0,1)$, there is a minimizer $u_{(a, b)} \in \Gamma_{I}$ of $\mathscr{E}_{\text {non' }}$,

$$
(-\Delta)^{s} u_{(a, b)}=b(x) f\left(u_{(a, b)}\right) \quad \text { in }(a, b)
$$

and $u_{(a, b)} \in C^{2, \alpha}(a, b)$ for some $0<\alpha<1$. 
Proof. We can change the values of $G$ for $|x|>1$ such that $G$ has the linear growth there which is denoted by $\widetilde{G}$. Given the admissible set

$$
\widetilde{\Gamma}_{I}=\left\{w \in H^{s}(I), w=-1 \text { for } x \leq a, w=1 \text { for } x \geq b\right\} .
$$

Clearly $\Gamma_{I} \subset \widetilde{\Gamma}_{I}$ and $h(x-((a+b) / 2))$ is an element of them. Consider the energy functional

$$
\begin{aligned}
\widetilde{\mathscr{E}}_{\text {non }}(w, I)= & \frac{1}{2} \iint_{I} \frac{|w(x)-w(y)|^{2}}{|x-y|^{1+2 s}} d y d x \\
& +\int_{I} \int_{\mathbb{R} \backslash I} \frac{|w(x)-w(y)|^{2}}{|x-y|^{1+2 s}} d y d x \\
& +\int_{I} b(x) \widetilde{G}(w) d x .
\end{aligned}
$$

If the minimizer $w$ of $\widetilde{\mathscr{E}}_{\text {non }}$ in $\widetilde{\Gamma}_{I}$ satisfies $|w| \leq 1$, then $w \in \Gamma_{I}$ and $\widetilde{\mathscr{E}}_{\text {non }}(w, I)=\mathscr{E}_{\text {non }}(w, I)$; that is, $w$ is also a minimizer of $\mathscr{E}_{\text {non }}$ in $\Gamma_{I}$. and

Obviously, the energy is nonincreasing by cutting $w$ at \pm 1

$$
\widetilde{\mathscr{E}}_{\mathrm{non}}(w, I)=\frac{1}{2}\|w\|_{\dot{H}^{s}(I)}^{2}+\int_{I} b(x) \widetilde{G}(w) d x
$$

is well defined, bounded below, and coercive in $\widetilde{\Gamma}_{I}$. Thus, there is a minimizer $u_{(a, b)} \in \Gamma_{I}$,

$$
\begin{aligned}
& (-\Delta)^{s} u_{(a, b)}=b(x) f\left(u_{(a, b)}\right) \quad \text { in }(a, b), \\
& \mathscr{E}_{\text {non }}\left(u_{(a, b)},(a, b)\right) \\
& \leq \begin{cases}C_{s}\left(1+|b-a|^{1-2 s}\right) & \text { if } s \in\left(0, \frac{1}{2}\right), \\
C_{s}(1+\log |b-a|) & \text { if } s=\frac{1}{2}, \\
C_{s} & \text { if } s \in\left(\frac{1}{2}, 1\right)\end{cases}
\end{aligned}
$$

by (37).

Lemma 10. Let $s \in(1 / 2,1)$. Let $u_{(0, R)}$ be the minimizer of $\mathscr{E}_{\text {non }}(\cdot,(0, R))$ with $R>4$; then

$$
\mathscr{E}_{\text {non }}\left(u_{(0, R)},(-l, l)\right) \leq \mathscr{E}_{\text {non }}\left(u_{(0, R)}+\phi,(-l, l)\right)+\alpha_{l}(R)
$$

for any $\phi \in C_{0}^{\infty}(-l, l)$ and $l \in Z^{+}$, where $\alpha_{l} \rightarrow 0$ as $l \rightarrow \infty$.

Proof. Denote $Z_{R}(x)=u_{(-l, R+l)}(((R+2 l) / R) x-l)$. Clearly, $Z_{R}=1$ as $x \geq R$ and $Z_{R}=-1$ as $x \leq 0$.
One has

$$
\begin{aligned}
& \mathscr{E}_{\text {non }}\left(Z_{R},(0, R)\right) \\
& =\frac{1}{2} \iint_{0}^{R}\left|u_{(-l, R+l)}\left(\frac{R+2 l}{R} x-l\right)-u_{(-l, R+l)}\left(\frac{R+2 l}{R} y-l\right)\right|^{2} \\
& \times\left(|x-y|^{1+2 s}\right)^{-1} \\
& +\int_{0}^{R} \int_{(-\infty, 0) \cup(R,+\infty)} \mid u_{(-l, R+l)}\left(\frac{R+2 l}{R} x-l\right) \\
& -\left.u_{(-l, R+l)}\left(\frac{R+2 l}{R} y-l\right)\right|^{2} \\
& \times\left(|x-y|^{1+2 s}\right)^{-1} \\
& +\int_{0}^{R} b(x) G\left(u_{(-l, R+l)}\left(\frac{R+2 l}{R} x-l\right)\right) \\
& =\left(\frac{R}{R+2 l}\right)^{1-2 s} \frac{1}{2} \iint_{-l}^{R+l} \frac{\left|u_{(-l, R+l)}(x)-u_{(-l, R+l)}(y)\right|^{2}}{|x-y|^{1+2 s}} \\
& +\left(\frac{R}{R+2 l}\right)^{1-2 s} \\
& \times \int_{-l}^{R+l} \int_{(-\infty,-l) \cup(R+l,+\infty)} \frac{\left|u_{(-l, R+l)}(x)-u_{(-l, R+l)}(y)\right|^{2}}{|x-y|^{1+2 s}} \\
& +\frac{R}{R+2 l} \int_{-l}^{R+l} b\left(\frac{R}{R+2 l}(x+l)\right) G\left(u_{(-l, R+l)}\right) \\
& \leq\left(\frac{R}{R+2 l}\right)^{1-2 s} E_{\text {non }}\left(u_{(-l, R+l)},(-l, R+l)\right) \\
& +\left(\frac{R}{R+2 l}\right)^{1-2 s} \\
& \times \int_{-l}^{R+l}\left\{b\left(\frac{R}{R+2 l}(x+l)\right)-b(x)\right\} G\left(u_{(-l, R+l)}\right) .
\end{aligned}
$$

Denote

$$
\begin{aligned}
\beta_{l}(R)= & \left(\frac{R}{R+2 l}\right)^{1-2 s} \\
& \times \int_{-l}^{R+l}\left\{b\left(\frac{R}{R+2 l}(x+l)\right)-b(x)\right\} G\left(u_{(-l, R+l)}\right) \\
\leq & \left(\frac{R}{R+2 l}\right)^{1-2 s} C_{s} \sup _{\mathbb{R}}\left|b\left(\left(\frac{R}{R+2 l}\right)(x+l)\right)-b(x)\right| \\
\longrightarrow & 0 ;
\end{aligned}
$$


here we use the periodic of $b$;

$$
\begin{aligned}
\gamma_{l}(R) & =\left\{\left(\frac{R}{R+2 l}\right)^{1-2 s}-1\right\} \mathscr{E}_{\text {non }}\left(u_{(-l, R+l)},(-l, R+l)\right) \\
& \leq C_{s}\left\{\left(\frac{R}{R+2 l}\right)^{1-2 s}-1\right\} \longrightarrow 0
\end{aligned}
$$

as $R \rightarrow \infty$.

Therefore,

$$
\begin{aligned}
\mathscr{E}_{\text {non }}\left(Z_{R},(0, R)\right) \leq & \mathscr{E}_{\text {non }}\left(u_{(-l, R+l)},(-l, R+l)\right) \\
& +\gamma_{l}(R)+\beta_{l}(R) .
\end{aligned}
$$

Since $\boldsymbol{u}_{(0, R)}$ is the minimizer of $\mathscr{E}_{\text {non }}(\cdot,(0, R))$,

$$
\begin{aligned}
\mathscr{E}_{\text {non }}\left(u_{(0, R)},(0, R)\right) \leq & \mathscr{E}_{\text {non }}\left(u_{(-l, R+l)},(-l, R+l)\right) \\
& +\gamma_{l}(R)+\beta_{l}(R) .
\end{aligned}
$$

Let

$$
\begin{aligned}
\eta_{l}(R)= & \mathscr{E}_{\text {non }}\left(u_{(0, R)},(-l, R+l)\right)-\mathscr{E}_{\text {non }}\left(u_{(0, R)},(0, R)\right) \\
= & \int_{-l}^{0} \int_{R}^{\infty} \frac{\left|u_{(0, R)}(x)-u_{(0, R)}(y)\right|^{2}}{|x-y|^{1+2 s}} \\
& +\int_{R}^{R+l} \int_{-\infty}^{-l} \frac{\left|u_{(0, R)}(x)-u_{(0, R)}(y)\right|^{2}}{|x-y|^{1+2 s}} \\
\leq & 4 \int_{-l}^{0} \int_{R}^{\infty}|x-y|^{-1-2 s} d y d x \\
& +4 \int_{R}^{R+l} \int_{-\infty}^{-l}|x-y|^{-1-2 s} d y d x \\
= & \frac{2}{s(2 s-1)}\left\{R^{1-2 s}-(R+2 l)^{1-2 s}\right\} \longrightarrow 0
\end{aligned}
$$

as $R \rightarrow \infty$.

By (48)-(49) and the fact that $u_{(-l, R+l)}$ is the minimizer in $(-l, R+l)$,

$$
\begin{aligned}
& \mathscr{E}_{\text {non }}\left(u_{(0, R)},(-l, R+l)\right) \\
& \leq \mathscr{E}_{\text {non }}\left(u_{(-l, R+l)},(-l, R+l)\right)+\gamma_{l}(R)+\beta_{l}(R)+\eta_{l}(R) \\
& \leq \mathscr{C}_{\text {non }}\left(u_{(0, R)}+\phi,(-l, R+l)\right)+\gamma_{l}(R)+\beta_{l}(R)+\eta_{l}(R) ; \\
& \mathscr{E}_{\text {non }}\left(u_{(0, R)}+\phi,(-l, R+l)\right)-\mathscr{C}_{\mathrm{non}}\left(u_{(0, R)},(-l, R+l)\right) \\
&=\frac{1}{2} \iint_{-l}^{l} \frac{\left|\left(u_{(0, R)}+\phi\right)(x)-\left(u_{(0, R)}+\phi\right)(y)\right|^{2}}{|x-y|^{1+2 s}} \\
& \quad-\frac{1}{2} \iint_{-l}^{l} \frac{\left|u_{(0, R)}(x)-u_{(0, R)}(y)\right|^{2}}{|x-y|^{1+2 s}} \\
& \quad+\int_{-l}^{l} \int_{l}^{+\infty} \frac{\left|\left(u_{(0, R)}+\phi\right)(x)-\left(u_{(0, R)}+\phi\right)(y)\right|^{2}}{|x-y|^{1+2 s}}
\end{aligned}
$$

$$
\begin{aligned}
& -\int_{-l}^{l} \int_{l}^{+\infty} \frac{\left|u_{(0, R)}(x)-u_{(0, R)}(y)\right|^{2}}{|x-y|^{1+2 s}} \\
& +\int_{-l}^{l} \int_{-\infty}^{-l} \frac{\left|\left(u_{(0, R)}+\phi\right)(x)-\left(u_{(0, R)}+\phi\right)(y)\right|^{2}}{|x-y|^{1+2 s}} \\
& -\int_{-l}^{l} \int_{-\infty}^{-l} \frac{\left|u_{(0, R)}(x)-u_{(0, R)}(y)\right|^{2}}{|x-y|^{1+2 s}} \\
& +\int_{-l}^{l} b(x)\left\{G\left(u_{(0, R)}+\phi\right)-G\left(u_{(0, R)}\right)\right\} \\
& =\mathscr{E}_{\text {non }}\left(u_{(0, R)}+\phi,(-l, l)\right)-\mathscr{E}_{\text {non }}\left(u_{(0, R)},(-l, l)\right) .
\end{aligned}
$$

Therefore,

$$
\mathscr{E}_{\text {non }}\left(u_{(0, R)},(-l, l)\right) \leq \mathscr{E}_{\text {non }}\left(u_{(0, R)}+\phi,(-l, l)\right)+\alpha_{l}(R),
$$

where $\alpha_{l}(R)=\gamma_{l}(R)+\beta_{l}(R)+\eta_{l}(R) \rightarrow 0$ as $R \rightarrow \infty$. The proof of Lemma 10 is completed.

It is noticed that $\left\|u_{(0, R)}\right\|_{C^{2, \alpha}} \leq C$ for some $C$ independent of $R$; then by the canonical diagonal process, up to a subsequence, $u_{(0, R)} \rightarrow u_{\infty}$ in $C_{\mathrm{loc}}^{2}(\mathbb{R})$ as $R \rightarrow \infty$ and

$$
\begin{aligned}
\left(-\partial_{x x}\right)^{s} u_{\infty} & =b(x) f\left(u_{\infty}\right) \quad \text { in }(0, \infty), \\
u_{\infty} & =-1 \quad \text { in }(-\infty, 0] .
\end{aligned}
$$

For $u_{\infty}$, we have the following.

Lemma 11. $u_{\infty}$ is a local minimizer of the nonlocal energy; that is, for every $l>0$ and for any $\phi \in C_{0}^{\infty}(-l, l)$,

$$
\mathscr{E}_{\text {non }}\left(u_{\infty},(-l, l)\right) \leq \mathscr{E}_{\text {non }}\left(u_{\infty}+\phi,(-l, l)\right) .
$$

Proof. Since $u_{(0, R)} \rightarrow u_{\infty}$ in $C_{\text {loc }}^{2}(\mathbb{R})$,

$$
\begin{array}{r}
\frac{1}{2} \iint_{-l}^{l} \frac{\left|u_{(0, R)}(x)-u_{(0, R)}(y)\right|^{2}}{|x-y|^{1+2 s}} \\
\longrightarrow \frac{1}{2} \iint_{-l}^{l} \frac{\left|u_{\infty}(x)-u_{\infty}(y)\right|^{2}}{|x-y|^{1+2 s}}, \\
\int_{-l}^{l} b(x) G\left(u_{(0, R)}\right)-\int_{-l}^{l} b(x) G\left(u_{\infty}\right) .
\end{array}
$$

For $|y| \geq 2 l$ and $|x| \leq l,|x-y| \geq|y|-|x| \geq|y| / 2$,

$$
\begin{aligned}
& \int_{-l}^{l} \int_{\mathbb{R} \backslash(-2 l, 2 l)} \frac{\left|u_{(0, R)}(x)-u_{(0, R)}(y)\right|^{2}}{|x-y|^{1+2 s}} \\
& \quad \leq 4 \int_{-l}^{l} \int_{\mathbb{R} \backslash(-2 l, 2 l)}|x-y|^{-1-2 s} \\
& \quad \leq 4 \int_{-l}^{l} d x \int_{\mathbb{R} \backslash(-2 l, 2 l)} \frac{2^{1+2 s}}{|y|^{1+2 s}} d y \leq \frac{16}{s} l^{1-2 s} .
\end{aligned}
$$


Thus, by the Dominated convergence theorem and locally uniformly convergence,

$$
\begin{aligned}
\int_{-l}^{l} \int_{\mathbb{R} \backslash(-l, l)} \frac{\left|u_{(0, R)}(x)-u_{(0, R)}(y)\right|^{2}}{|x-y|^{1+2 s}} \\
\quad=\int_{-l}^{l} \int_{\mathbb{R} \backslash(-2 l, 2 l)} \frac{\left|u_{(0, R)}(x)-u_{(0, R)}(y)\right|^{2}}{|x-y|^{1+2 s}} \\
\quad+\int_{-l}^{l} \int_{(-2 l, 2 l) \backslash(-l, l)} \frac{\left|u_{(0, R)}(x)-u_{(0, R)}(y)\right|^{2}}{|x-y|^{1+2 s}} \\
\longrightarrow \int_{-l}^{l} \int_{\mathbb{R} \backslash(-l, l)} \frac{\left|u_{\infty}(x)-u_{\infty}(y)\right|^{2}}{|x-y|^{1+2 s}} .
\end{aligned}
$$

By (54) and the above,

$$
\mathscr{E}_{\text {non }}\left(u_{(0, R)},(-l, l)\right) \longrightarrow \mathscr{E}_{\text {non }}\left(u_{\infty},(-l, l)\right)
$$

as $R \rightarrow \infty$. The same discussion leads to the fact that

$$
\mathscr{E}_{\text {non }}\left(u_{(0, R)}+\phi,(-l, l)\right) \longrightarrow \mathscr{E}_{\text {non }}\left(u_{\infty}+\phi,(-l, l)\right)
$$

as $R \rightarrow \infty$ and for any $\phi \in C_{0}^{\infty}(-l, l)$.

Therefore, (53) follows from Lemma 10, and

$$
\begin{gathered}
\left(-\partial_{x x}\right)^{s} u_{\infty}=b(x) f\left(u_{\infty}\right), \quad-1 \leq u_{\infty} \leq 1 \quad \text { in } \mathbb{R}, \\
u_{\infty}=-1 \quad \text { in }(-\infty, 0] .
\end{gathered}
$$

Furthermore $u_{\infty} \equiv-1$ by the strong maximum principle.

Let $u_{(-R, R)}$ be the minimizer of $\mathscr{E}_{\text {non }}(\cdot,(-R, R))$ in $\Gamma_{(-R, R)}$. By continuity, there is a $P_{R}=\sup \left\{x \in(-R, R), u_{(-R, R)} \leq 0\right\}$ such that $u_{(-R, R)}\left(P_{R}\right)=0$ and $u_{(-R, R)}>0$ for $x>P_{R}$.

Lemma 12. One has $R-\left|P_{R}\right| \rightarrow \infty$ as $R \rightarrow \infty$.

Proof. To check this, we suppose by contradiction that $\mid R+$ $P_{R} \mid<+\infty$ for some sequence $R \rightarrow \infty$. Up to a subsequence, $R+P_{R} \rightarrow k$ as $R \rightarrow \infty$ for some constant $k$.

One has

$$
-1 \longleftarrow u_{(0,2 R)}(x)=u_{(-R, R)}(x-R)>0
$$

for $x>k$ and $R$ large enough. This contradiction verifies our conclusion.

Now we start to prove our conclusion.

Proof of Theorem 3. Let $u^{R}(x)=u_{(-R, R)}\left(x+\left[P_{R}\right]\right)$ for $x \in$ $\left(-R-\left[P_{R}\right], R-\left[P_{R}\right]\right)$, where $\left[P_{R}\right]$ is denoted by the integer part of $P_{R}$. And $u^{R}(x)$ satisfies

$$
(-\Delta)^{s} u^{R}=b(x) f\left(u^{R}\right) \quad \text { in }\left(-R-\left[P_{R}\right], R-\left[P_{R}\right]\right) .
$$

Up to a subsequence which is denoted again by $u^{R}, u^{R} \rightarrow u$ in $C_{\text {loc }}^{2}(\mathbb{R})$ as $R \rightarrow \infty$ and

$$
(-\Delta)^{s} u=b(x) f(u) \text { in }(-\infty, \infty) .
$$

Since $u^{R}\left(P_{R}-\left[P_{R}\right]\right)=u_{(-R, R)}\left(P_{R}\right)=0$ and $0 \leq P_{R}-\left[P_{R}\right]<1$, for the above subsequence, there is a sub-subsequence such that $P_{R}-\left[P_{R}\right] \rightarrow P \in[0,1], u(P)=0$, and $u \geq 0$ for $x \geq P$.

One has

$$
\begin{aligned}
\mathscr{E}_{\text {non }} & \left(u^{R},\left(-R-\left[P_{R}\right], R-\left[P_{R}\right]\right)\right) \\
= & \mathscr{E}_{\text {non }}\left(u_{(-R, R)},(-R, R)\right) \leq C_{s} .
\end{aligned}
$$

By Fatou's lemma and Lemma $12, \mathscr{E}_{\text {non }}(u, \mathbb{R}) \leq C_{s}$ and $\int_{-\infty}^{+\infty} b(x) G(u)<+\infty$.

We claim that $|u| \rightarrow \pm 1$ as $|x| \rightarrow \infty$. Indeed, if there exists a sequence $x_{n} \rightarrow \infty$ such that $\liminf _{x \rightarrow \infty}|u|=$ $\lim _{x_{n} \rightarrow \infty}\left|u\left(x_{n}\right)\right| \neq 1$, by the $C^{2}$ regularity of $u$, there must be a ball $B_{\rho}\left(x_{n}\right)$ such that $G(u)>\epsilon$ in $B_{\rho}\left(x_{n}\right)$ for some $\epsilon>0$,

$$
\begin{aligned}
\infty & >\int_{-\infty}^{+\infty} b(x) G(u) \geq \sum_{n=1}^{\infty} \int_{B_{\rho}\left(z_{n}\right)} b(x) G(u(x)) \\
& \geq \underline{b} \cdot \epsilon \sum_{n=1}^{\infty} 2 \rho=\infty .
\end{aligned}
$$

Therefore, $u \rightarrow 1$ or -1 as $x \rightarrow \infty$. The proof as $x \rightarrow-\infty$ is similar. Since $u \geq 0$ for $x>P \in[0,1], u \rightarrow 1$ as $x \rightarrow \infty$.

In the following we exclude the case that $u \rightarrow 1$ as $x \rightarrow$ $-\infty$.

Assume that $u \rightarrow 1$ as $x \rightarrow-\infty$ by contradiction. Denote $C_{0}=\min _{R} u=u\left(x_{0}\right)$ for some $x_{0} \in R$. $C_{0}>-1$ by the strong maximum principle.

One has

$$
\int_{\mathbb{R}} \frac{u\left(x_{0}\right)-u(y)}{|x-y|^{1+2 s}} d y=(-\Delta)^{s} u\left(x_{0}\right)=b\left(x_{0}\right) f\left(u\left(x_{0}\right)\right)<0,
$$

$f\left(u\left(x_{0}\right)\right)=f\left(C_{0}\right)<0$. Therefore $C_{0} \notin[1-\delta, 1]$ by Remark 4 . Choose a small positive constant $\delta^{*}<\min \{\delta, 1-$ $\left.\left(\left(\left|C_{0}\right|+1\right) / 2\right)\right\}$ such that $G\left(1-\delta^{*}\right)<\min \{G(1-\delta), G(-1+$ $\left.\delta), G\left(-\left(\left|C_{0}\right|+1\right) / 2\right)\right\}$. Denote $S=\inf \left\{x \in \mathbb{R}, u<1-\delta^{*}\right\}$ and $O=\sup \left\{x \in R, u<1-\delta^{*}\right\} ; S$ and $O$ are well defined since $u \rightarrow \pm 1$ as $x \rightarrow \pm \infty$.

Denote

$$
\begin{aligned}
T_{R} & =\inf \left\{x \in(-R, R), u_{(-R, R)} \geq 1-\delta^{*}\right\}, \\
S_{R} & =\inf \left\{x \in\left(T_{R}, R\right), u_{(-R, R)}<1-\delta^{*}\right\}, \\
O_{R} & =\sup \left\{x \in(-R, R), u_{(-R, R)}<1-\delta^{*}\right\}, \\
C_{0, R} & =\min _{\left(S_{R}, O_{R}\right)} u_{(-R, R)}=u_{(-R, R)}\left(x_{R}\right), \\
Q_{R} & =\inf \left\{x>P_{R}, u_{(-R, R)}=\frac{\left|C_{0}\right|+1}{2}\right\} .
\end{aligned}
$$


If $R$ is large enough, $T_{R}, S_{R}, O_{R}, C_{0, R}, x_{R}$, and $Q_{R}$ are all well defined. By local uniform convergence, $\left|C_{0, R}\right| \in$ $\left(\left|C_{0}\right| / 2,\left(\left|C_{0}\right|+1\right) / 2\right)$ and $\left|O_{R}-S_{R}\right|<|O-S|+1=M$ :

$$
\begin{aligned}
\frac{\left|C_{0}\right|+1}{2} & =\left|u_{(-R, R)}\left(P_{R}\right)-u_{(-R, R)}\left(Q_{R}\right)\right| \\
& \leq\left\|u_{(-R, R)}\right\|_{C^{2, \beta}(R)}\left|P_{R}-Q_{R}\right| \leq C\left|P_{R}-Q_{R}\right|
\end{aligned}
$$

for some positive constant $C$ independent of $R,\left|P_{R}-Q_{R}\right| \geq$ $L>0$.

For simplicity, denote $I_{1}=\left[-R, T_{R}\right], I_{2}=\left[T_{R}, S_{R}\right], I_{3}=$ $\left[S_{R}, O_{R}\right]$, and $I_{4}=\left[O_{R}, R\right]$; then $[-R, R]=\cup_{i=1}^{4} I_{i}$.

Define

$$
u_{R}^{*}= \begin{cases}1-\delta^{*} & \text { if } x \in I_{3} \cap\left\{u_{(-R, R)}<1-\delta^{*}\right\}=I_{3}^{*}, \\ u_{(-R, R)} & \text { if elsewhere. }\end{cases}
$$

We compare the energy of $u_{R}^{*}$ and of $u_{(-R, R)}$ :

$$
\begin{gathered}
\mathscr{E}_{\text {non }}\left(u_{(-R, R)},(-R, R)\right)-\mathscr{E}_{\text {non }}\left(u_{R}^{*},(-R, R)\right) \\
=\frac{1}{2} \int_{I_{1} \cup I_{2} \cup I_{3} \cup I_{4}} \int_{I_{3}^{*}}\left\{\frac{\left|u_{(-R, R)}(x)-u_{(-R, R)}(y)\right|^{2}}{|x-y|^{1+2 s}}\right. \\
\left.-\frac{\left|u_{R}^{*}(x)-u_{R}^{*}(y)\right|^{2}}{|x-y|^{1+2 s}}\right\} \\
+\int_{I_{3}^{*}} \int_{(-\infty,-R) \cup(R,+\infty)}\left\{\frac{\left|u_{(-R, R)}(x)-u_{(-R, R)}(y)\right|^{2}}{|x-y|^{1+2 s}}\right. \\
\left.-\frac{\left|u_{R}^{*}(x)-u_{R}^{*}(y)\right|^{2}}{|x-y|^{1+2 s}}\right\} \\
+\int_{I_{3}^{*}} b(x)\left\{G\left(u_{(-R, R)}\right)-G\left(u_{R}^{*}\right)\right\} .
\end{gathered}
$$

Since $u \rightarrow 1$ as $x \rightarrow \pm \infty,\left|O_{R}-T_{R}\right|>\left|S_{R}-T_{R}\right| \rightarrow \infty$ as $R \rightarrow \infty$.

One has

$$
\begin{aligned}
\frac{1}{2} \int_{I_{1}} \int_{I_{3}^{*}} \frac{\left|u_{(-R, R)}(x)-u_{(-R, R)}(y)\right|^{2}}{|x-y|^{1+2 s}} & \\
& +\int_{I_{3}^{*}} \int_{-\infty}^{-R} \frac{\left|u_{(-R, R)}(x)-u_{(-R, R)}(y)\right|^{2}}{|x-y|^{1+2 s}} \\
& -\frac{1}{2} \int_{I_{1}} \int_{I_{3}^{*}} \frac{\left|u_{R}^{*}(x)-u_{R}^{*}(y)\right|^{2}}{|x-y|^{1+2 s}} \\
& -\int_{I_{3}^{*}} \int_{-\infty}^{-R} \frac{\left|u_{R}^{*}(x)-u_{R}^{*}(y)\right|^{2}}{|x-y|^{1+2 s}} \\
\geq & -4 \int_{-\infty}^{T_{R}} \int_{I_{3}} \frac{1}{|x-y|^{1+2 s}} d y \\
= & -\frac{2}{s(2 s-1)}\left(\left|S_{R}-T_{R}\right|^{1-2 s}-\left|O_{R}-T_{R}\right|^{1-2 s}\right) \longrightarrow 0
\end{aligned}
$$

as $R \rightarrow \infty$.
Since $u_{(-R, R)} \geq 1-\delta^{*}$ in $I_{2} \cup I_{4} \cup\left(I_{3} \backslash I_{3}^{*}\right)$ and $u_{(-R, R)}<1-\delta^{*}$ in $I_{3}^{*}$,

$$
\begin{aligned}
& \frac{1}{2} \int_{I_{2} \cup I_{4} \cup\left(I_{3} \backslash I_{3}^{*}\right)} \int_{I_{3}^{*}}\left(\frac{\left|u_{(-R, R)}(x)-u_{(-R, R)}(y)\right|^{2}}{|x-y|^{1+2 s}}\right. \\
& \left.-\frac{\left|u_{(-R, R)}(x)-u_{R}^{*}(y)\right|^{2}}{|x-y|^{1+2 s}}\right) \\
& +\int_{I_{3}^{*}} \int_{R}^{\infty} \frac{\left|u_{(-R, R)}(x)-1\right|^{2}}{|x-y|^{1+2 s}}-\int_{I_{3}^{*}} \int_{R}^{\infty} \frac{\left|u_{R}^{*}(y)-1\right|^{2}}{|x-y|^{1+2 s}} \geq 0, \\
& \frac{1}{2} \iint_{I_{3}^{*}} \frac{\left|u_{(-R, R)}(x)-u_{(-R, R)}(y)\right|^{2}}{|x-y|^{1+2 s}} \\
& -\frac{1}{2} \iint_{I_{3}^{*}} \frac{\left|u_{R}^{*}(x)-u_{R}^{*}(y)\right|^{2}}{|x-y|^{1+2 s}} \\
& >0 \text {, } \\
& \int_{I_{3}^{*}} b(x)\left\{G\left(u_{(-R, R)}\right)-G\left(u_{R}^{*}\right)\right\} \\
& \geq \underline{b} \int_{P_{R}}^{Q_{R}}\left\{G\left(u_{(-R, R)}\right)-G\left(u_{R}^{*}\right)\right\} \\
& \geq \underline{b} L\left\{\min \left(G(1-\delta), G(-1+\delta), G\left(-\frac{1+\left|C_{0}\right|}{2}\right)\right)\right. \\
& \left.-G\left(1-\delta^{*}\right)\right\}
\end{aligned}
$$$$
>0 .
$$

Therefore, $\mathscr{E}_{\text {non }}\left(u_{(-R, R)},(-R, R)\right)>\mathscr{E}_{\text {non }}\left(u_{R}^{*},(-R, R)\right)$ for $R$ large enough. This contradiction deduces the fact that $u \rightarrow$ -1 as $x \rightarrow-\infty . u$ is the desired solution.

\section{Appendix}

In this appendix we state several regularity results involving the nonlocal equation (1), the local equation (3), and a Hopf lemma; their proof can be found in $[2,3,6]$.

Proposition A.1. Let $b \in C^{1, \gamma}(\mathbb{R}) \cap L^{\infty}(\mathbb{R})$ and $f \in C^{1, \gamma}(\mathbb{R})$ with $\gamma>\max (0,1-2 s)$. Then, any bounded solution of (1)

$$
(-\Delta)^{s} v(x)=b\left(x_{1}\right) f(v(x)) \quad \text { in } \mathbb{R}^{n}
$$

is $C^{2, \beta}\left(\mathbb{R}^{n}\right)$ for some $0<\beta<1$ depending only on s and $\gamma$.

Furthermore, given $1 / 2<s_{0}$ there exists $0<\beta<1$ depending only on $n, s_{0}$, and $\gamma$ and hence independent of $s-$ such that, for every $s>s_{0}$,

$$
\|v\|_{C^{2, \beta}\left(\mathbb{R}^{n}\right)} \leq C
$$


for some $C$ depending only on $n, s_{0},\|f\|_{C^{1, \gamma}},\|b\|_{C^{1, \gamma},}$, and $\|v\|_{L^{\infty}}$, not on $s$. In addition, the function $u=P_{s} * v$, where $P_{s}$ is the Poisson kernel $\left(u(x, y)=p_{n, s} \int_{\mathbb{R}^{n}}\left(y^{2 s} /\left(z^{2}+y^{2}\right)^{n+2 s}\right) v(x-z) d z\right)$, satisfies

$$
\|u\|_{C^{\beta}\left(\overline{\mathbb{R}_{+}^{n+1}}\right)}+\left\|\nabla_{x} u\right\|_{C^{\beta}\left(\overline{\mathbb{R}_{+}^{n+1}}\right)}+\left\|D_{x}^{2} u\right\|_{C^{\beta}\left(\overline{\mathbb{R}_{+}^{n+1}}\right)} \leq C .
$$

If $s>s_{0}$, the constant $C$ is independent of $s$ and only dependent on the quantities as the above mentioned.

We remark that the above results are valid for solutions of (A.1) in bounded domains.

Proposition A.2. Let $a \in(-1,1)$ and $R>0$. Let $\varphi \in$ $C^{\sigma}\left(\partial^{0} B_{2 R}^{+}\right)$for some $\sigma \in(0,1)$ and $u \in L^{\infty}\left(B_{2 R}^{+}\right) \cap H^{1}\left(B_{2 R}^{+}, y^{a}\right)$ be a weak solution of

$$
\begin{array}{cc}
-\operatorname{div}\left(y^{a} \nabla u(x, y)\right)=0 \quad \text { in } B_{2 R}^{+} \subset \mathbb{R}_{+}^{n+1}, \\
-\lim _{y \downarrow 0^{+}} y^{a} u_{y}(x, y)=\varphi & \text { on } \partial^{0} B_{2 R}^{+} .
\end{array}
$$

Then, there exists $\beta \in(0,1)$ depending only on $n, a, R$, $\|u\|_{L^{\infty}\left(B_{2 R}^{+}\right)}$, and also $\|\varphi\|_{L^{\infty}\left(\partial^{0} B_{2 R}^{+}\right)}\left(\right.$for $\left.C_{R}^{1}\right)$ and $\|\varphi\|_{C^{\sigma}\left(\partial^{0} B_{2 R}^{+}\right)}($for $C_{R}^{2}$ ), such that

$$
\begin{gathered}
\|u\|_{C^{0, \beta}\left(\overline{B_{R}^{+}}\right)} \leq C_{R}^{1}, \\
\left\|y^{a} u_{y}\right\|_{C^{0, \beta}\left(\overline{B_{R}^{+}}\right)} \leq C_{R}^{2} .
\end{gathered}
$$

Lemma A.3 (Hopf Principle). Let $a \in(-1,1)$ and consider the cylinder $C_{R, 1}=\Gamma_{R}^{0} \times(0,1) \subset \mathbb{R}_{+}^{n+1}$ where $\Gamma_{R}^{0}$ is the ball of center 0 and radius $R$ in $\mathbb{R}^{n}$. Let $u \in C\left(\overline{C_{R, 1}}\right) \cap H^{1}\left(C_{R, 1}, y^{a}\right)$ satisfy

$$
\begin{gathered}
\operatorname{div}\left(y^{a} \nabla u(x, y)\right) \leq 0 \quad \text { in } C_{R, 1} \\
u>0 \quad \text { in } C_{R, 1}, \\
u(0,0)=0 .
\end{gathered}
$$

Then,

$$
\limsup _{y \downarrow 0^{+}}-y^{a} \frac{u(0, y)}{y}<0
$$

In addition, if $y^{a} u_{y} \in C\left(\overline{C_{R, 1}}\right)$, then

$$
\lim _{y \downarrow 0^{+}} y^{a} u_{y}(0, y)<0 .
$$

\section{Conflict of Interests}

The author declares that there is no conflict of interests regarding the publication of this paper.

\section{Acknowledgment}

This research has been supported by National Natural Science Foundation of China (Grant no. 11371128).

\section{References}

[1] X. Cabré and J. Solà-Morales, "Layer solutions in a half-space for boundary reactions," Communications on Pure and Applied Mathematics, vol. 58, no. 12, pp. 1678-1732, 2005.

[2] X. Cabré and Y. Sire, Nonlinear Equations for Fractional Laplacians, I: Regularity, Maximum Principles, and Hamiltonian Estimates, Annales de l'Institut Henri Poincare, Non Linear Analysis, Elsevier Masson, 2013.

[3] X. Cabre and Y. Sire, "Nonlinear equations for fractional Laplacians II: existence, uniqueness, and qualitative properties of solutions," http://arxiv.org/abs/1111. 0796.

[4] L. Caffarelli and L. Silvestre, "An extension problem related to the fractional laplacian," Communications in Partial Differential Equations, vol. 32, no. 8, pp. 1245-1260, 2007.

[5] E. B. Fabes, C. E. Kenig, and R. P. Serapioni, “The local regularity of solutions of degenerate elliptic equations," Communications in Statistics-Theory and Methods, vol. 7, no. 1, pp. 77-116, 1982.

[6] G. Palatucci, O. Savin, and E. Valdinoci, "Local and global minimizers for a variational energy involving a fractional norm," Annali di Matematica Pura ed Applicata, vol. 192, no. 4, pp. 673-718, 2013.

[7] S. Alama, L. Bronsard, and C. Gui, "Stationary layered solutions in $\mathbb{R}^{2}$ for an Allen-Cahn system with multiple well potential," Calculus of Variations and Partial Differential Equations, vol. 5, no. 4, pp. 359-390, 1997.

[8] N. Ghoussoub and C. Gui, "On a conjecture of De Giorgi and some related problems," Mathematische Annalen, vol. 311, no. 3, pp. 481-491, 1998.

[9] N. Ghoussoub and C. Gui, "On De Giorgi's conjecture in dimensions 4 and 5," Annals of Mathematics, vol. 157, no. 1, pp. 313-334, 2003.

[10] Y. Hu, "Layer solutions for a class of semilinear elliptic equations involving fractional Laplacians," Boundary Value Problems, vol. 2014, article 41, 2014.

[11] R. A. Adams and J. J. F. Fournier, Sobolev Spaces, Academic Press, 2003.

[12] D. Gilbarg and N. S. Trudinger, Elliptic Partial Differential Equations of Second Order, Springer, 2001.

[13] A. Nekvinda, "Characterization of traces of the weighted Sobolev space $W^{1, p}\left(\Omega, d_{M}^{\epsilon}\right)$ on $M$,' Czechoslovak Mathematical Journal, vol. 43, no. 4, pp. 695-711, 1993. 


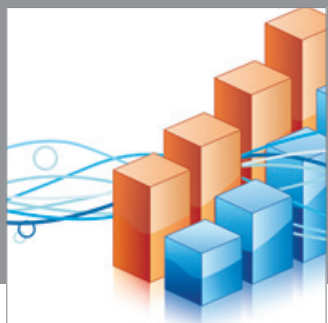

Advances in

Operations Research

mansans

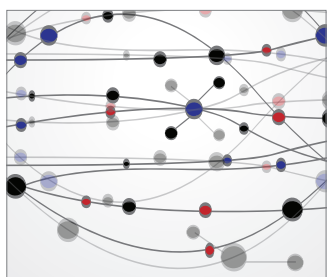

The Scientific World Journal
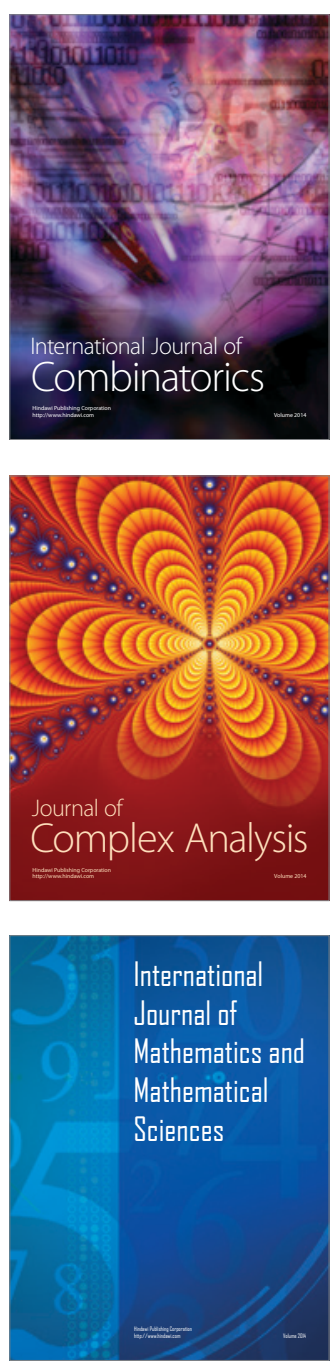
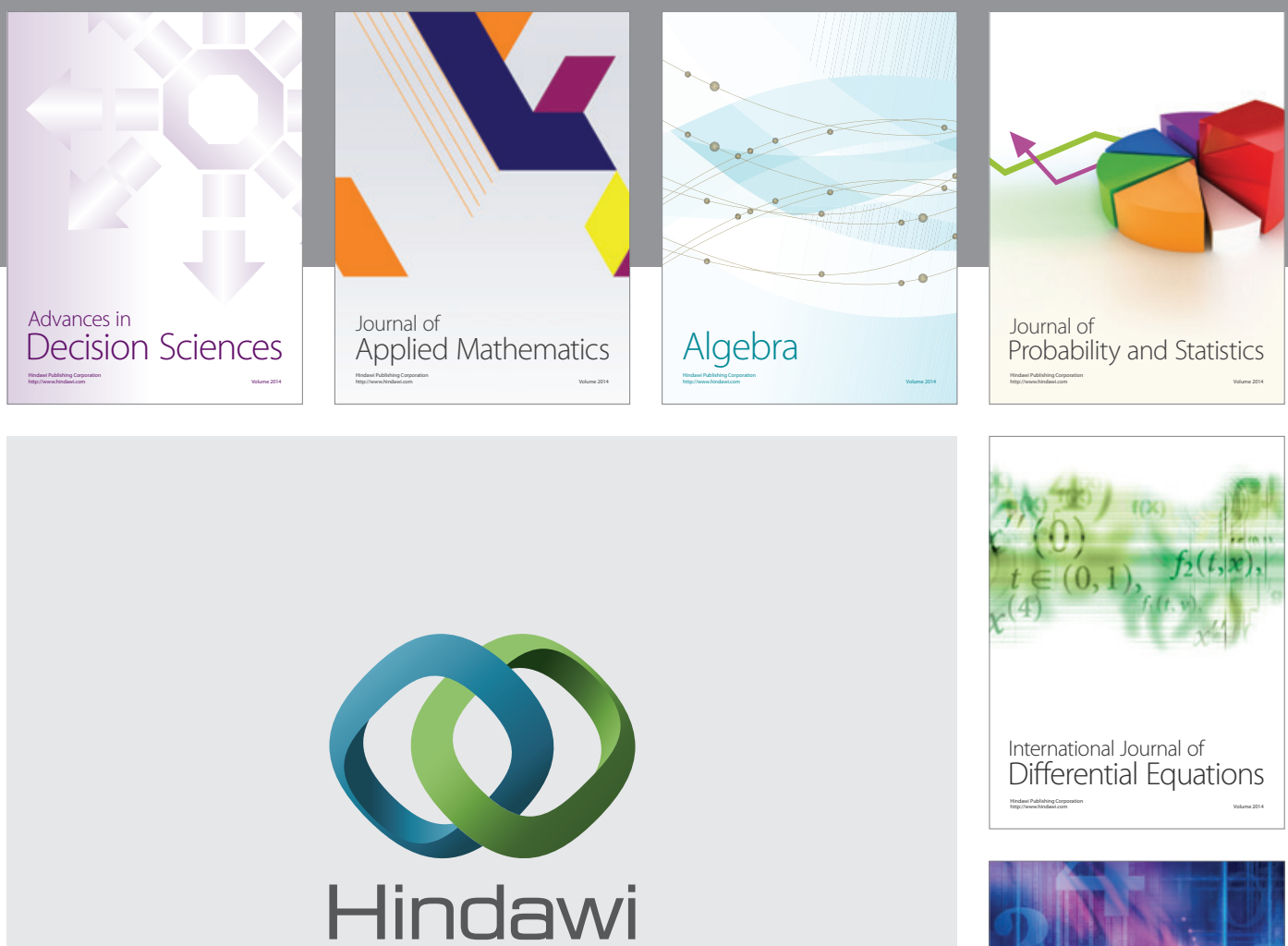

Submit your manuscripts at http://www.hindawi.com
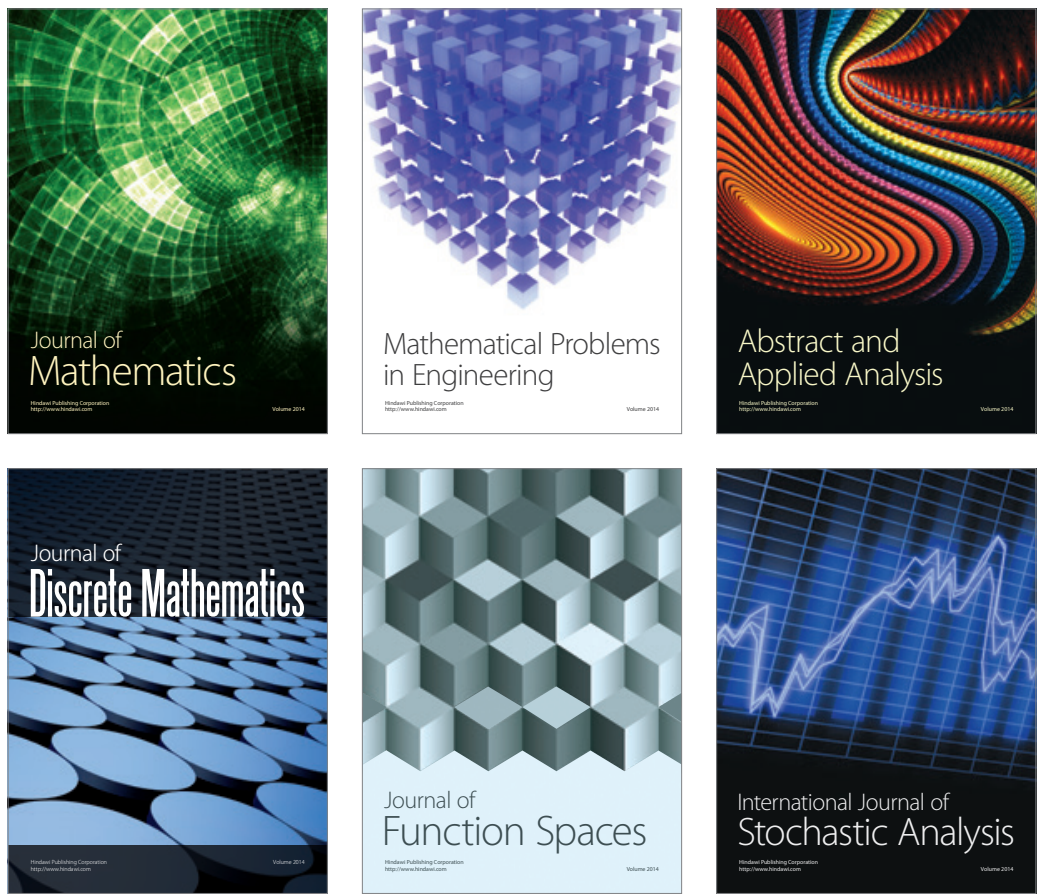

Journal of

Function Spaces

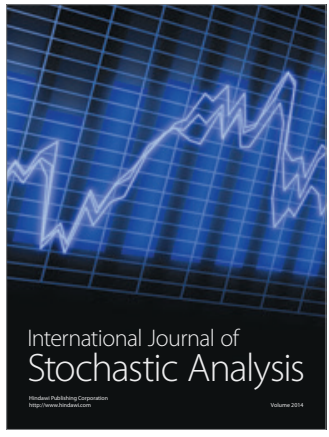

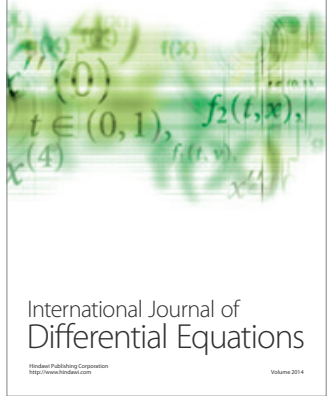
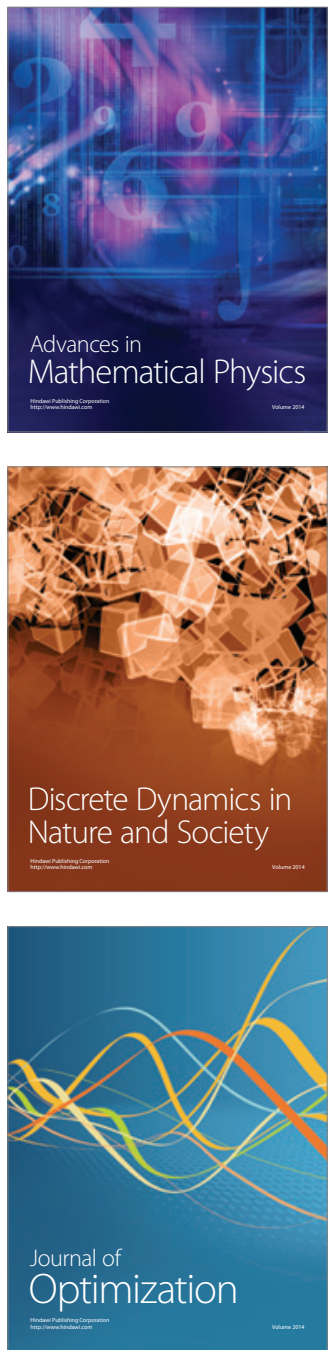